\title{
Embryonal tumor with multilayered rosettes of the fourth ventricle: case report
}

\author{
Catherine A. Edmonson, BSc, ${ }^{1}$ Kristin J. Weaver, MD, PhD, ${ }^{1}$ Jesse Kresak, MD, ${ }^{2}$ and \\ David W. Pincus, MD, PhD'
}

Departments of ${ }^{1}$ Neurosurgery and ${ }^{2}$ Pathology, University of Florida, Gainesville, Florida

\begin{abstract}
Embryonal tumor with multilayered rosettes (ETMR) is a recently described pathological entity. These primitive central nervous system tumors harbor amplification of the 19q13.42 locus and resultant overexpression of the LIN28A protein. Although the WHO currently recognizes 3 distinct histopathological entities-embryonal tumor with abundant neuropil and true rosettes (ETANTR), ependymoblastoma, and medulloepithelioma-recent studies indicate that these tumors have a common molecular profile and clinical course and that they are now classified as a single entity. Here the authors present a case of ETMR located in the fourth ventricle in a 12-month-old boy. The histopathology featured areas of neuropil-like stroma and highly cellular foci with characteristic multilayered rosettes. The authors discuss the clinical, radiological, and histopathological findings in this case and compare them with data in previously published cases in the literature. A review of studies assessing the molecular mechanisms underlying these tumors is also presented.
\end{abstract}

http://thejns.org/doi/abs/10.3171/2015.3.PEDS1525

KEY WORDS embryonal tumor; ETANTR; fourth ventricle tumor; microRNA; LIN28A; ETMR; oncology

$\mathrm{C}$ ENTRAL nervous system primitive neuroectodermal tumors (PNETs) are most commonly diagnosed in children and are associated with a variable prognosis. ${ }^{20}$ One histological subtype, termed "embryonal tumor with abundant neuropil and true rosettes" (ETANTR), compose a small subset of CNS PNETs that are poorly understood. Since Eberhart et al.'s original description of this subtype in 2000, fewer than 100 cases of ETANTR have been reported. ${ }^{7}$ More recently it has been discovered that ETANTR, along with 2 other CNS PNET variants known as medulloepithelioma and ependymoblastoma, share a common molecular phenotype and clinical course, and thus are now collectively classified as embryonal tumor with multilayered rosettes (ETMR). Here we present a case of ETMR, specifically an ETANTR, arising from the fourth ventricle, which has only been documented once before. ${ }^{2}$ We describe the clinical, pathological, and radiographic features of this rare tumor and review the relevant literature of ETMR.

\section{Case Report}

History and Initial Presentation

A previously healthy 12-month-old boy presented to an outside regional hospital emergency room with a 2-week history of "increased wobbliness" when sitting up. Complete blood count and metabolic panel obtained at the outside hospital were within normal limits. Head CT showed a large mass in the posterior fossa measuring approximately $4.4 \times 4.5 \mathrm{~cm}$ and obstructive hydrocephalus. Upon presentation to our institution, the patient's mother reported that the child seemed "more unstable on his feet lately" even though he was an experienced walker and that he had been "fussier" lately with decreased appetite. His birth, medical, surgical, and family histories were not significant. The child was developmentally appropriate for his age and was up to date on his immunizations.

Physical exam upon presentation revealed an alert but irritable child. Vital signs were within normal limits. His

ABBREVIATIONS AT/RT = atypical teratoid/rhabdoid tumor; ETANTR $=$ embryonal tumor with abundant neuropil and true rosettes; ETMR $=$ embryonal tumor with multilayered rosettes; $\mathrm{EVD}=$ external ventricular drain; FISH = fluorescence in situ hybridization; $\mathrm{HGG}$ = high-grade glioma; miRNA = microRNA; PNET = primitive neuroectodermal tumor; POD = postoperative day.

SUBMITTED January 13, 2015. ACCEPTED March 31, 2015.

INCLUDE WHEN CITING Published online August 7, 2015; DOI: 10.3171/2015.3.PEDS1525. 
anterior fontanelle was open, nonbulging, and $3 \mathrm{~mm}$ in width and his head circumference was $54 \mathrm{~cm}$. Neurological exam revealed normal muscle tone in upper and lower extremities with no sign of atrophy. No gross cranial nerve deficits were apparent. Sensation was preserved bilaterally in the upper and lower extremities. Coordination examination was significant for ataxic movement in the upright position. Deep tendon reflexes were preserved in the upper and lower extremities without pathological reflexes.

Given the head CT findings, the patient was electively intubated for placement of an external ventricular drain (EVD) followed by MRI of the entire neuraxis. Magnetic resonance imaging confirmed a $5.8 \times 4.7 \times 4.7-\mathrm{cm}$ solid mass in the fourth ventricle causing compression of the fourth ventricle and obstructive hydrocephalus. The mass was hypointense on T1-weighted imaging and hyperintense on T2-weighted imaging and showed heterogeneous enhancement within the mass (Fig. 1). No metastatic deposits were visualized.

\section{Operation}

A suboccipital craniotomy for tumor resection was performed. On gross examination of the mass, the tissue appeared soft, fleshy, and pink-white in color. Gross-total excision was achieved and the aqueduct was visualized with good CSF flow. A specimen was sent for frozen section and diagnosis at this time was embryonal tumor, likely medulloblastoma as opposed to atypical teratoid/rhabdoid tumor (AT/RT).
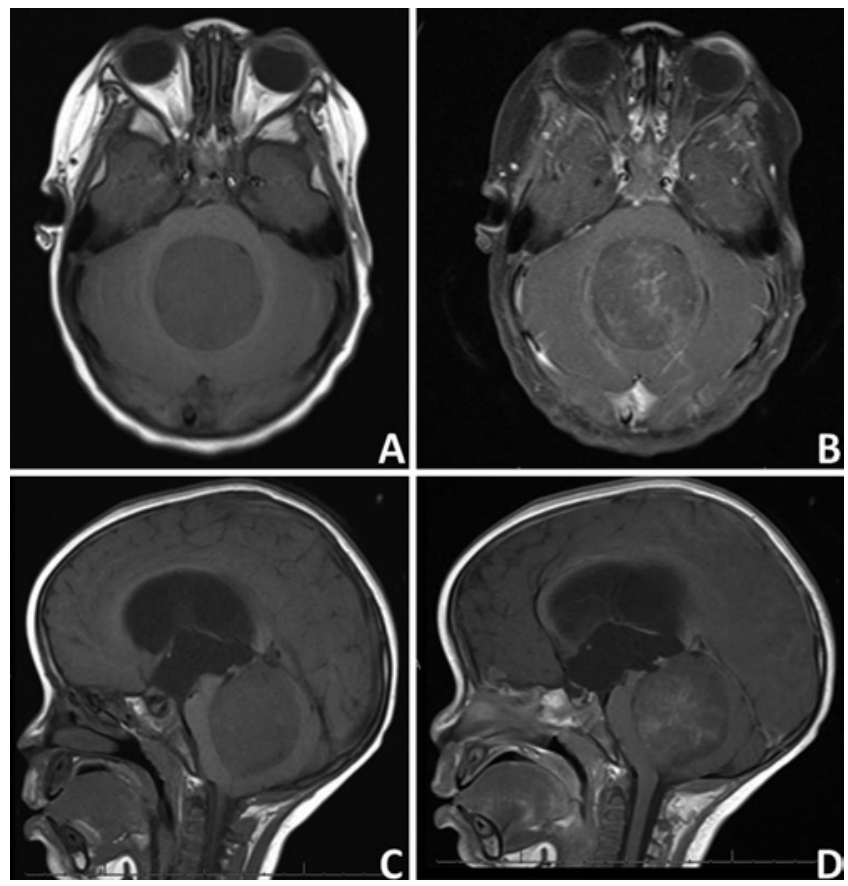

FIG. 1. Axial (A) and sagittal (C) T1-weighted MR images showing a hypointense $5.8 \times 4.7 \times 4.7-\mathrm{cm}$ solid mass in the fourth ventricle compressing the brainstem. Compression of the pituitary from a dilated third ventricle is visible. There is mild heterogeneous enhancement of the mass on axial (B) and sagittal (D) Gd-enhanced T1-weighted MR images.
Histopathological Examination

Histological analysis of the resected specimen showed a biphasic tumor with hypercellular areas of small blue cells with minimal cytoplasm (reminiscent of medulloblastoma/PNET) admixed with paucicellular neuropil areas (Fig. 2). Additionally numerous multilayered rosettes were found in both regions of the tumor. Immunohistochemical staining for neurofilament protein was diffusely positive, while synaptophysin highlighted the PNET and neuropil areas but was negative in the rosettes. Glial fibrillary acidic protein and NeuN were negative throughout. Immunohistochemical study for INI-1 showed retained nuclear staining in the tumor cells. Chromosome 19 expression studies were not available at our institution.

\section{Postoperative Course}

Magnetic resonance imaging performed on postoperative Day (POD) 1 revealed no evidence of residual tumor and expected postsurgical changes in the fourth ventricle (Fig. 3). His EVD was clamped and intracranial pressures were monitored. His intracranial pressure remained normal and his EVD was removed.

Chemotherapy consisting of vincristine, cisplatin, etoposide, cyclophosphamide, and methotrexate with leucovorin rescue was started on POD 18 according to the ACNS0333 protocol. Radiotherapy was not pursued since we believed that its short and long-term risks did not outweigh its benefits in a child his age. The patient's hospital

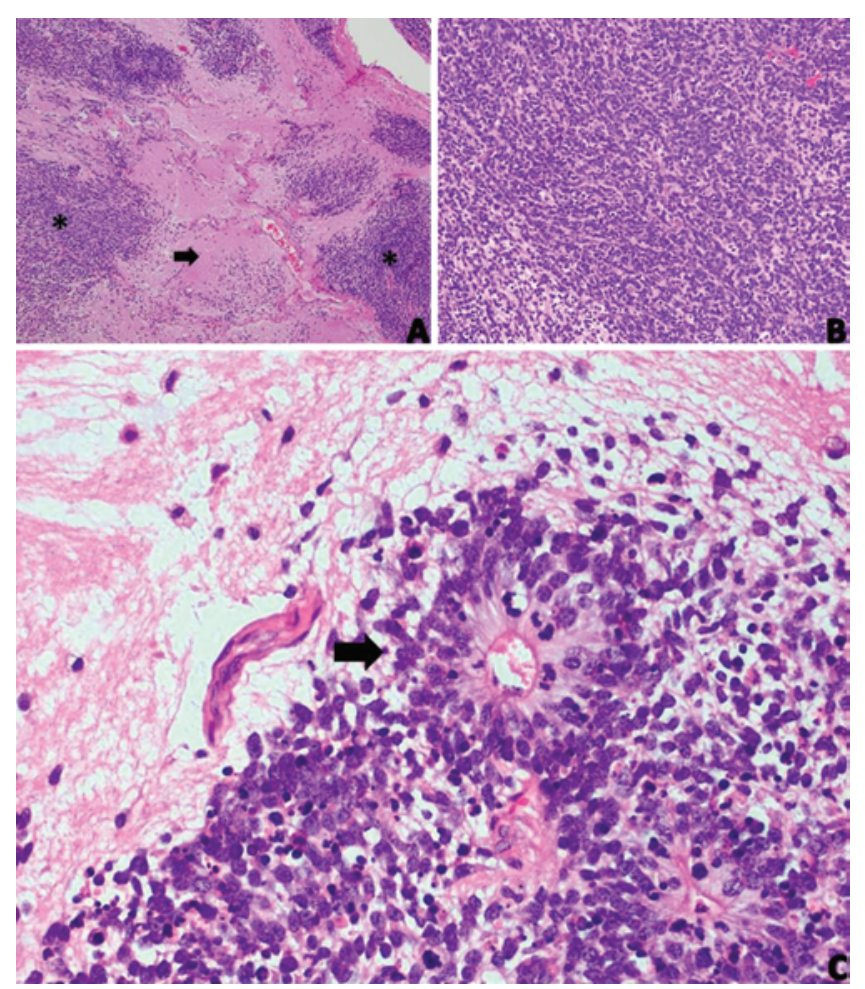

FIG. 2. A: Paucicellular neuropil (arrow) admixed with PNET-like areas containing multilayered rosettes (asterisks). Original magnification $\times 40$. B: Hypercellular embryonal areas of small blue cells with minimal cytoplasm. Original magnification $\times 100$. C: Arrow indicating multilayered true rosette with distinct lumen. $H \& E$, original magnification $\times 400$. Figure is available in color online only. 

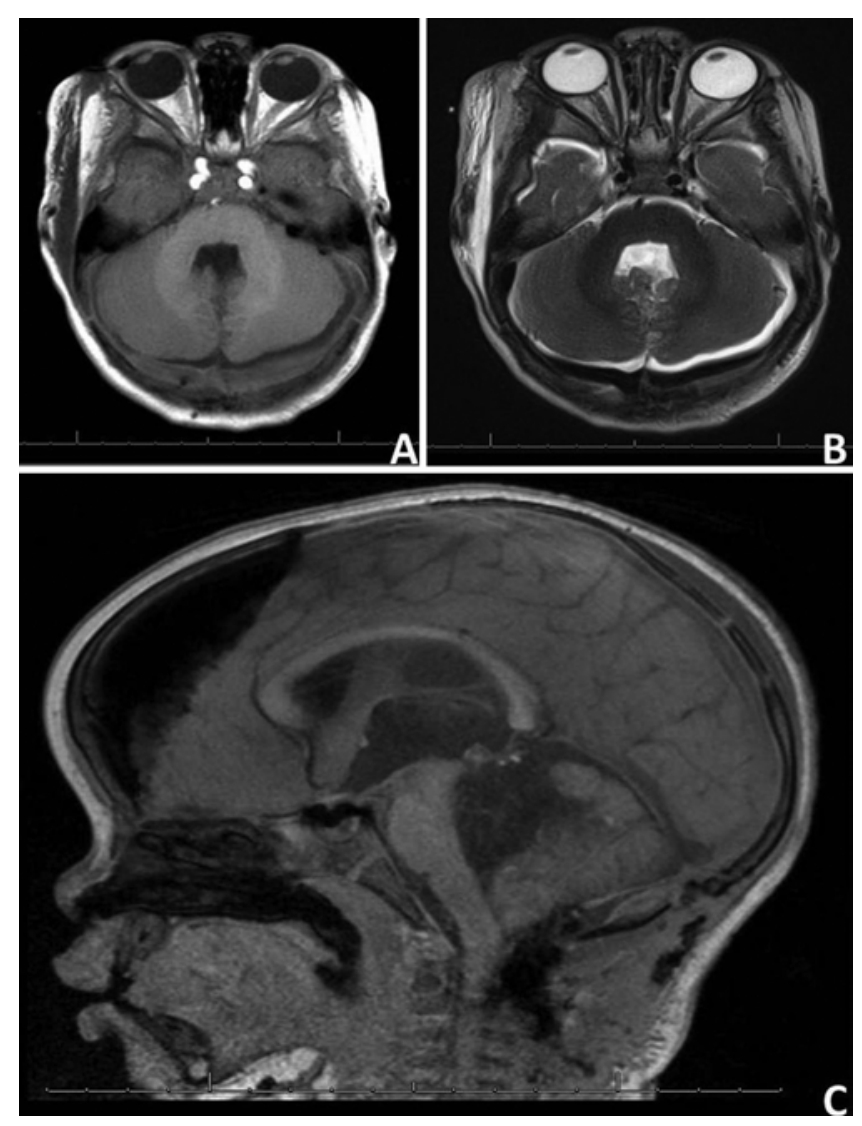

FIG. 3. Axial T1-weighted (A) and T2-weighted (B) MR images showing air and fluid in the postsurgical bed. Sagittal T1-weighted image (C) showing pneumocephalus in the frontal lobes with lateral and third ventricles decreased in size compared with their appearance preoperatively (Fig. 1C and D)

course was complicated by bacteremia with Enterococcus faecalis, coagulase-negative Staphylococcus sp., Candida albicans, and Candida glabrata and Clostridium difficile in stool. He was treated with appropriate antibiotics and antifungal agents. On POD 42, the patient developed a dysconjugate gaze with bilateral cranial nerve VI palsies, and repeat MRI revealed fourth ventricle outflow obstruction. He underwent placement of a ventriculoperitoneal shunt. Chemotherapy is ongoing.

\section{Discussion}

Embryonal tumors typically present in young children and adolescents and are often associated with an aggressive course. According to the 2007 WHO classification system, these tumors are classified as medulloblastomas (the most common type), AT/RTs, or CNS PNETs. ${ }^{20}$ Within CNS PNETs, there are 3 recognized histological variants: ETANTR, medulloepithelioma, and ependymoblastoma. Recent molecular studies have shown that these 3 tumor types harbor a common molecular phenotype and may in fact represent a histological spectrum that is now unified under the term "ETMR." We maintain the term "ETANTR" for the purposes of reviewing the literature since it was the term used in most previous studies.

ETANTR was first described in 2000 by Eberhart et al. ${ }^{7}$ In this seminal article these authors reported 7 cases of CNS PNETs, characterized histologically by undifferentiated neuroepithelial cells, areas of well-differentiated neuropil, and ependymoblastic rosettes arising from paucicellular regions of the neuropil. ${ }^{11}$ In 2009, a follow-up of this original report was published, which included 22 additional cases and follow-up on the original 7 cases. ${ }^{11}$ This updated report demonstrated that the majority of tumors were found in the supratentorial region and were predominately seen in females with a mean age of 2 years. ${ }^{11}$ The majority of patients showed little or no response to chemotherapy and radiation, with over $75 \%$ of patients dying within 30 months of initial presentation and the longest survival being only 42 months. ${ }^{11}$

Since the original report in 2000 , fewer than 100 additional cases of ETANTR have been reported in the literature. ${ }^{1-6,8-12,15-18,21,23,24,26-30}$ In the majority of cases, including those described in the original report, the tumor was found in the supratentorial region (65 total), whereas only 35 were found in the infratentorial region-16 in the brainstem and 1 in the spinal cord. There was a slight female predominance (52\%), with an average age of 28 months at initial presentation. Of the supratentorial tumors, most involved the parietal or frontal lobe. Two of the 65 supratentorial tumors invaded the dura mater, and 1 showed spinal metastasis at initial presentation.

Of the 35 infratentorial tumors reported in the literature, only 1 has been described as occurring in the fourth ventricle, as in the patient in the present case. ${ }^{2}$ Similar to the patient we report here, this other child with a fourth ventricle tumor underwent resection and was started on a treatment regimen that included 5 cycles of chemotherapy with vincristine, cisplatin, etoposide, cyclophosphamide, and methotrexate with leucovorin and stem cell rescue.

Sixteen tumors documented in the literature appeared in the brainstem, with 2 presenting with spinal metastasis at initial diagnosis. ${ }^{11,27}$ One tumor was exclusively found in the spinal cord - this patient did not undergo resection and died 6 months after presentation. ${ }^{11}$

The prognosis associated with ETANTRs is poor with a $69 \%$ mortality rate overall and more than $75 \%$ of these deaths occurring within a year of initial presentation. Of all reported cases, brainstem tumors are associated with the worst prognosis with only $7 \%$ of patients diseasefree at follow-up, although supratentorial and cerebellar tumor patients have a slightly better prognosis with $11 \%$ and $9 \%$ disease-free at follow-up, respectively. The worse prognosis is probably attributable to the fact that brainstem tumors are often unresectable because of their location, as compared with lesions in the cortex or cerebellum. Only $38 \%$ of tumors documented in the brainstem were resected (either completely or partially), compared with $72 \%$ of cerebellar tumors and $69 \%$ of supratentorial tumors. Notably, the longest reported survival for a patient with ETANTR without residual disease is 7 years. ${ }^{21}$ However, it is not clear whether other long-term survivors exist, as there is very limited long-term follow-up data on ETANTR patients in the literature, which has been attributed to the fact that most reported series are in pathology journals, and thus clinical follow-up is not discussed in detail. $^{21}$ 
Recent studies have attempted to elucidate the genetic and molecular profile of these tumors, with the goal of identifying tumor-specific markers that can be used for diagnostic, prognostic, and therapeutic purposes. The first cytogenetic studies of ETANTRs included a case with isochromosome 17, a finding typically seen only in medulloblastoma, and another case with tumor cells exhibiting polysomy of chromosomes $2,8,17$, and 22 on fluorescence in situ hybridization (FISH) analysis..$^{10}$ In 2009, using array comparative genomic hybridization, Pfister et al. reported a tumor that, in addition to exhibiting polysomy of chromosomes 2 and 19, showed amplification of a chromosome band at 19q13.42, which encompasses a cluster of microRNAs (miRNAs) denoted "C19MC." ${ }^{26}$ Interestingly, 2 of the miRNAs in this cluster, mir-372 and mir-373, have been implicated as oncogenes in the p53 regulatory pathway. ${ }^{28}$

Following this initial report of a copy number variation at this miRNA locus, Li et al. described amplification of this cluster in 11 additional cases of supratentorial PNETs and hypothesized that this amplification might be associated with ependymoblastic differentiation. ${ }^{19}$ To test this hypothesis, Korshunov et al. performed FISH analysis on 41 tumor samples histologically diagnosed as ependymoblastomas and ETANTRs and found that $97 \%$ of the samples showed amplification at the 19q13.42 locus, suggesting that ETANTRs and ependymoblastomas may not be separate entities. ${ }^{16}$ Subsequently, multiple other reports have demonstrated amplification of this region in tumors with ependymoblastic rosettes. In 2010, Paulus and Kleihues coined the term "embryonal tumor with multilayered rosettes." $24,25,29$

After the original report by Pfister et al. showing chromosome 19 amplification in ETMRs, other studies documented molecular abnormalities in these tumors. ${ }^{2,5}$ In 2012, Korshunov et al. proposed LIN28A overexpression as a potential biomarker for ETMRs after finding by immunohistochemistry that $100 \%$ of the ETMRs they analyzed were $L I N 28 A$ positive compared with only $12 \%$ (6/50) of the AT/RTs and none of the 41 CNS PNETs (non-ETMR variants), 334 medulloblastomas, 223 anaplastic ependymomas, and 131 pediatric glioblastomas. ${ }^{16}$ LIN28A and its homolog LIN28B encode proteins that bind and repress the let-7 family of miRNAs. They are thought to act as oncogenes when overexpressed via let7 repression and subsequent upregulation of let-7 targets such as $M Y C$ and $R A S .^{13,22}$ Similarly, a study by Spence et al., which included 128 medulloblastomas, 45 AT/RTs, 105 ependymomas, 50 high-grade gliomas (HGGs), 20 choroid plexus carcinomas, and 103 CNS PNETs, revealed that amplification at 19q13.42 was observed only in CNS PNETs, whereas LIN28A immunoreactivity was observed in $19.5 \%$ of HGGs and $24.4 \%$ of AT/RTs in addition to a subset of CNS PNETs. ${ }^{27}$ Together these studies suggest that LIN28A positivity may be sensitive to, but not specific for, ETMR. Conversely, 19q13.42 amplification appears to be specific for ETMRs. ${ }^{27}$

In a similar analysis, Korshunov et al. evaluated 97 tumors from patients diagnosed with ETANTR, ependymoblastoma, or medulloepithelioma and demonstrated that these tumors could not be distinguished from each other at a molecular level based on 19q13.42 amplification and LIN28A positivity. Thus, they corroborated previous suggestions that these tumors should be considered a single diagnostic entity. ${ }^{17}$ Additionally, they noted that these tumors share a similar clinical course, typically occurring in children younger than 3 years of age, usually supratentorial in origin, and associated with a poor prognosis. ${ }^{17}$

Most recently, Kleinman et al. looked at the methylation patterns of ETMRs, hypothesizing that the miRNA cluster amplification seen in these tumors may be affecting global methylation patterns..$^{14}$ Interestingly, they reported that $D N M T 3 B$, a methyltransferase involved in DNA methylation and thought to be important in embryonic differentiation, was overexpressed in the 12 ETMRs they studied as compared with other tumors in The Cancer Genome Atlas, making overexpression of this protein another potential diagnostic biomarker. Moreover, exon 1B of DNMT3B is predominately expressed during Week 8 of fetal development and was only found to be expressed in ETMRs, suggesting that aberrant splicing during neurogenesis may result in tumor formation. Future work should attempt to define the normal expression of this miRNA cluster and its methylation pattern over early human neurodevelopment, in a manner similar to the Kleinman et al. study of $D N M T 3 B$, as perhaps dysregulation of expression of this miRNA cluster early in development drives subsequent molecular and histopathological changes seen in these tumors.

\section{Conclusions}

ETANTRs, now belonging to the classification ETMRs, are a group of rare tumors usually found in the supratentorial region in young children. Treatment of this tumor includes resection, chemotherapy, and radiotherapy in most cases, although even with appropriate treatment the prognosis is poor. Molecular studies have recently identified amplification of the C19MC miRNA cluster and overexpression of LIN28A in these tumors, suggesting a potential molecular mechanism for the pathogenesis of this cancer. Furthermore, such findings may prove useful as diagnostic biomarkers for this tumor and potential targetable therapies. Future work concentrating on understanding the normal function of the C19MC miRNA cluster in human brain development may lead to a better understanding of the molecular pathogenesis of ETMRs and identify additional molecular biomarkers for targetable therapies and prognostic utility in these aggressive tumors.

\section{References}

1. Adamek D, Sofowora KD, Cwiklinska M, Herman-Sucharska I, Kwiatkowski S: Embryonal tumor with abundant neuropil and true rosettes: an autopsy case-based update and review of the literature. Childs Nerv Syst 29:849-854, 2013

2. Alexiou GA, Stefanaki K, Vartholomatos G, Sfakianos G, Prodromou N, Moschovi M: Embryonal tumor with abundant neuropil and true rosettes: a systematic literature review and report of 2 new cases. J Child Neurol 28:1709-1715, 2013

3. Al-Hussain TO, Dababo MA: Posterior fossa tumor in a 2 year-old girl. Brain Pathol 19:343-346, 2009

4. Al-Hussaini M, Abuirmeileh N, Swaidan M, Al-Jumaily U, Rajjal H, Musharbash A, et al: Embryonal tumor with abundant neuropil and true rosettes: a report of three cases of a 
rare tumor, with an unusual case showing rhabdomyoblastic and melanocytic differentiation. Neuropathology 31:620625,2011

5. Buccoliero AM, Castiglione F, Rossi Degl'Innocenti D, Franchi A, Paglierani M, Sanzo M, et al: Embryonal tumor with abundant neuropil and true rosettes: morphological, immunohistochemical, ultrastructural and molecular study of a case showing features of medulloepithelioma and areas of mesenchymal and epithelial differentiation. Neuropathology 30:84-91, 2010

6. Dunham C, Sugo E, Tobias V, Wills E, Perry A: Embryonal tumor with abundant neuropil and true rosettes (ETANTR): report of a case with prominent neurocytic differentiation. J Neurooncol 84:91-98, 2007

7. Eberhart CG, Brat DJ, Cohen KJ, Burger PC: Pediatric neuroblastic brain tumors containing abundant neuropil and true rosettes. Pediatr Dev Pathol 3:346-352, 2000

8. Ferri Niguez B, Martínez-Lage JF, Almagro MJ, Fuster JL, Serrano C, Torroba MA, et al: Embryonal tumor with abundant neuropil and true rosettes (ETANTR): a new distinctive variety of pediatric PNET: a case-based update. Childs Nerv Syst 26:1003-1008, 2010

9. Frassanito P, D’Angelo L, Massimi L, Lauriola L, Novello M, Rocco CD, et al: Sudden paraplegia in a case of apparently isolated frontal embryonal tumour with abundant neuropil and true rosettes. Br J Neurosurg 26:284-286, 2012

10. Fuller C, Fouladi M, Gajjar A, Dalton J, Sanford RA, Helton KJ: Chromosome 17 abnormalities in pediatric neuroblastic tumor with abundant neuropil and true rosettes. Am J Clin Pathol 126:277-283, 2006

11. Gessi M, Giangaspero F, Lauriola L, Gardiman M, Scheithauer BW, Halliday W, et al: Embryonal tumors with abundant neuropil and true rosettes: a distinctive CNS primitive neuroectodermal tumor. Am J Surg Pathol 33:211-217, 2009

12. Hervey-Jumper SL, Altshuler DB, Wang AC, He X, Maher $\mathrm{CO}$, Robertson PL, et al: The role of CD133+ cells in a recurrent embryonal tumor with abundant neuropil and true rosettes (ETANTR). Brain Pathol 24:45-51, 2014

13. Johnson SM, Grosshans H, Shingara J, Byrom M, Jarvis R, Cheng A, et al: RAS is regulated by the let-7 microRNA family. Cell 120:635-647, 2005

14. Kleinman CL, Gerges N, Papillon-Cavanagh S, Sin-Chan P, Pramatarova A, Quang DA, et al: Fusion of TTYH1 with the C19MC microRNA cluster drives expression of a brainspecific DNMT3B isoform in the embryonal brain tumor ETMR. Nat Genet 46:39-44, 2014

15. Korshunov A, Remke M, Gessi M, Ryzhova M, Hielscher T, Witt H, et al: Focal genomic amplification at 19q13.42 comprises a powerful diagnostic marker for embryonal tumors with ependymoblastic rosettes. Acta Neuropathol 120:253260, 2010

16. Korshunov A, Ryzhova M, Jones DT, Northcott PA, van Sluis $\mathrm{P}$, Volckmann R, et al: LIN28A immunoreactivity is a potent diagnostic marker of embryonal tumor with multilayered rosettes (ETMR). Acta Neuropathol 124:875-881, 2012

17. Korshunov A, Sturm D, Ryzhova M, Hovestadt V, Gessi M, Jones DT, et al: Embryonal tumor with abundant neuropil and true rosettes (ETANTR), ependymoblastoma, and medulloepithelioma share molecular similarity and comprise a single clinicopathological entity. Acta Neuropathol 128:279-289, 2014

18. La Spina M, Pizzolitto S, Skrap M, Nocerino A, Russo G, Di Cataldo A, et al: Embryonal tumor with abundant neuropil and true rosettes. A new entity or only variations of a parent neoplasms (PNETs)? This is the dilemma. J Neurooncol 78:317-320, 2006

19. Li M, Lee KF, Lu Y, Clarke I, Shih D, Eberhart C, et al: Frequent amplification of a chr19q13.41 microRNA polycistron in aggressive primitive neuroectodermal brain tumors. Cancer Cell 16:533-546, 2009

20. Louis DN, Ohgaki H, Wiestler OD, Cavenee WK, Burger PC, Jouvet A, et al: The 2007 WHO classification of tumours of the central nervous system. Acta Neuropathol 114:97-109, 2007

21. Manjila S, Ray A, Hu Y, Cai DX, Cohen ML, Cohen AR: Embryonal tumors with abundant neuropil and true rosettes: 2 illustrative cases and a review of the literature. Neurosurg Focus 30(1):E2, 2011

22. Molenaar JJ, Domingo-Fernández R, Ebus ME, Lindner S, Koster J, Drabek K, et al: LIN28B induces neuroblastoma and enhances MYCN levels via let-7 suppression. Nat Genet 44:1199-1206, 2012

23. Nobusawa S, Orimo K, Horiguchi K, Ikota H, Yokoo H, Hirato J, et al: Embryonal tumor with abundant neuropil and true rosettes with only one structure suggestive of an ependymoblastic rosette. Pathol Int 64:472-477, 2014

24. Nobusawa S, Yokoo H, Hirato J, Kakita A, Takahashi H, Sugino T, et al: Analysis of chromosome 19q13.42 amplification in embryonal brain tumors with ependymoblastic multilayered rosettes. Brain Pathol 22:689-697, 2012

25. Paulus W, Kleihues P: Genetic profiling of CNS tumors extends histological classification. Acta Neuropathol 120:269-270, 2010

26. Pfister S, Remke M, Castoldi M, Bai AHC, Muckenthaler MU, Kulozik A, et al: Novel genomic amplification targeting the microRNA cluster at 19q13.42 in a pediatric embryonal tumor with abundant neuropil and true rosettes. Acta Neuropathol 117:457-464, 2009

27. Spence T, Sin-Chan P, Picard D, Barszczyk M, Hoss K, Lu $\mathrm{M}$, et al: CNS-PNETs with C19MC amplification and/or LIN28 expression comprise a distinct histogenetic diagnostic and therapeutic entity. Acta Neuropathol 128:291-303, 2014

28. Voorhoeve PM, le Sage C, Schrier M, Gillis AJM, Stoop H, Nagel R, et al: A genetic screen implicates miRNA-372 and miRNA-373 as oncogenes in testicular germ cell tumors. Cell 124:1169-1181,2006

29. Wang Y, Chu SG, Xiong J, Cheng HX, Chen H, Yao XH: Embryonal tumor with abundant neuropil and true rosettes (ETANTR) with a focal amplification at chromosome 19q13.42 locus: further evidence of two new instances in China. Neuropathology 31:639-647, 2011

30. Woehrer A, Slave I, Peyrl A, Czech T, Dorfer C, Prayer D, et al: Embryonal tumor with abundant neuropil and true rosettes (ETANTR) with loss of morphological but retained genetic key features during progression. Acta Neuropathol 122:787-790, 2011

\section{Disclosure}

The authors report no conflict of interest concerning the material or methods used in this study or the findings specified in this paper.

\section{Author Contributions}

Conception and design: Pincus. Acquisition of data: Edmonson. Analysis and interpretation of data: Weaver, Edmonson, Pincus. Drafting the article: Edmonson. Critically revising the article: all authors. Reviewed submitted version of manuscript: Weaver, Kresak, Pincus. Approved the final version of the manuscript on behalf of all authors: Weaver. Study supervision: Weaver, Pincus.

\section{Correspondence}

Kristin J. Weaver, Department of Neurosurgery, University of Florida, P.O. Box 100265, Gainesville, FL 32610. email: kristin. weaver@neurosurgery.ufl.edu. 